\title{
Influência dos parâmetros geométricos de rodovias na frequência e severidade de acidentes viários em curvas: o caso da BR 116
}

\author{
César Luís Andriola ${ }^{1}$, Tânia Batistela Torres ${ }^{2}$, Daniel Sergio Presta García ${ }^{3}$ \\ ${ }^{1}$ Laboratório de Sistemas de Transportes, UFRGS, andriola.cesar@gmail.com \\ 2Laboratório de Sistemas de Transportes, UFRGS, batistela.torres@ufrgs.br \\ 32aboratório de Sistemas de Transportes, UFRGS, daniel.garcia@ufrgs.br
}

\section{Recebido:}

29 de fevereiro de 2019

Aceito para publicação:

8 de julho de 2019

Publicado:

12 de novembro de 2019

Editor de área:

Flávio Cunto

\section{Palavras-chaves: \\ Segurança Viária, \\ Reconstituição Geométrica, \\ Parâmetros Geométricos, \\ Binomial Negativo, \\ Logit Ordenado.}

\section{Keywords:}

Road Safety,

Geometric Reconstitution,

Geometric Parameters,

Negative Binomial,

Ordered Logit.

DOI:10.14295/transportes.v27i3.1997

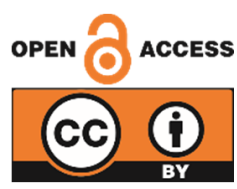

\begin{abstract}
RESUMO
Dada a necessidade de prover rodovias mais seguras aos usuários, este trabalho buscou identificar os impactos dos parâmetros geométricos na frequência e na severidade dos acidentes em curvas de rodovias de pista simples. Esta análise foi conduzida para 225 quilômetros de trechos rodoviários de pista simples da BR 116 no estado do Rio Grande do Sul. A reconstituição geométrica rodoviária viabilizou a construção de uma base de dados que reúne as características dos acidentes registrados entre 2007 e 2016 pela Polícia Rodoviária Federal (PRF) e os parâmetros geométricos, permitindo a modelagem da frequência e da severidade dos acidentes a partir desses parâmetros. Foram estimados os modelos econométricos: (i) binomial negativo (BN) para a modelagem da frequência; e (ii) logit ordenado (LO) para a severidade de acidentes. Os resultados indicam que o aumento da declividade e da superelevação impactam no aumento da frequência de acidentes e na redução da severidade.
\end{abstract}

\section{ABSTRACT}

Given the need to provide safer roads to users, this paper sought to identify the impact of geometrical parameters on the frequency and severity of accidents on curves in twolane highway. This analysis was carried out on 225 kilometers (circa 140 miles) of BR 116 two-lane highway stretches in the state of Rio Grande do Sul. Road geometrical reconstruction made possible the build of 2007-2016 registered accidents characteristics database by Federal Highway Police (PRF) and the geometrical parameters, allowing accidents frequency and severity modeling through these parameters. Two econometric models were estimated: (i) negative binomial (NB) for frequency modeling; and (ii) ordered logit $(\mathrm{OL})$ for accidents' severity. The results show that the increase in downward slope and superelevations contribute to the increase of accidents' frequency, though these helped on reducing its severity.

\section{INTRODUÇÃO}

Diante da possibilidade de os acidentes de trânsito causarem a perda de até 1,9 milhão de vidas por ano até 2020, governos de todo o mundo se comprometeram a tomar medidas para prevenir os acidentes de trânsito através do Plano de Ação Global para a Década (2011 a 2020) (WHO, 2011). Tais objetivos foram incorporados às diretrizes de Desenvolvimento Sustentável, através da Agenda 2030 (United Nations, 2017), de forma que o desenvolvimento deve ser pautado nos pilares de segurança: gestão de segurança viária, rodovias e mobilidade seguras, veículos seguros, usuários seguros e resposta à acidentes (United Nations, 2011). 
Os acidentes viários resultam de três fatores contribuintes: usuário, veículo e via (Treat et al., 1979). As pessoas cometem erros e são fisicamente vulneráveis em acidentes, enquanto que o sistema viário, da maneira como ainda é estruturado, contribui para que esses erros gerem acidentes (ITF, 2016). Nesse sentido, um projeto rodoviário seguro permite compensar, em parte, os riscos oferecidos pelos outros fatores, contribuindo com a redução da frequência dos acidentes e minimizando a severidade daqueles que ocorrerem.

Embora a maior parte dos acidentes em rodovias aconteça em trechos retos, são em curvas que ocorrem acidentes com maior severidade (Radimsky et al, 2016). Diversos estudos demonstram a necessidade de atualizar as atuais diretrizes para o projeto de curvas horizontais, principalmente no que se refere a trechos com elevadas declividades. 0 manual para projeto geométrico de rodovias vigente nos Estados Unidos (AASHTO, 2011) possui recomendações quanto ao projeto de curvas em grandes declividades, ao contrário do que ocorre no manual vigente para as rodovias federais brasileiras (DNER, 1999), que não aborda o tema.

Diversos estudos vêm abordando a frequência e ou a severidade de acidentes em rodovias, identificando os parâmetros geométricos que potencialmente as impactam. Uma série de estudos é dedicada à análise de rodovias de pista simples utilizando modelagens estatísticas e buscando determinar a influência de parâmetros geométricos na frequência de acidentes. Nesses estudos foram identificadas, na medida em que os valores desses parâmetros aumentam, a influência do ângulo central no aumento (Aram, 2010; Dong et al., 2015), do desenvolvimento no aumento (Aram, 2010; Schneider et al, 2010), do raio na redução (Schneider et al, 2010; Ma e Li, 2010; Hosseinpour et al., 2016; Cruz et al., 2017) e da superelevação no aumento da frequência de acidentes (Aram, 2010).

Também foram identificados autores que buscaram analisar os diferentes números de faixas ou tipos de veículos, e conjuntamente, buscaram determinar a influência dos parâmetros geométricos na frequência de acidentes. Esses estudos indicam que, na medida em que os valores desses parâmetros aumentam, a influência do ângulo central no aumento (Rengarasu et al, 2009; Garnaik, 2014), da declividade no aumento (Agbelie, 2016b; Shankar et al, 1995), do raio na redução (Garnaik, 2014; Iyinam et al., 1997) e da superelevação no aumento da frequência de acidentes (Garnaik, 2014). Outras variáveis encontradas como influentes foram a velocidade no aumento (Dong et al., 2015), a iluminação na redução (Agbelie, 2016a; Dong et al., 2015), a condição climática desfavorável no aumento (Shankar et al, 1995) e a redução da faixa etária do condutor no aumento da frequência de acidentes (De Oña et al, 2011).

Os estudos que analisaram a influência de parâmetros geométricos na severidade de acidentes em rodovias de pista simples identificaram a influência apenas do raio na redução da severidade de acidentes (Schneider et al., 2009). Outras variáveis encontradas como relevantes foram a velocidade e influência de álcool (Ma et al., 2015), que foram associadas ao aumento da severidade de acidentes. A presença de poucos estudos sobre a severidade de acidentes em rodovias de pista simples, especificamente, se dá por essa análise geralmente ser conduzida a partir de variáveis ambientais e do usuário.

Há, ainda, outros estudos que consideram diferentes números de faixas ou tipos de veículos, mas que também buscaram determinar a influência dos parâmetros geométricos na severidade de acidentes. Esses estudos indicam, na medida em que os valores desses parâmetros aumentam, a influência da declividade no aumento (Anastasopoulos et al., 2012; Chikkakrishna et al, 2017), do raio na redução (Anastasopoulos et al., 2012) e da superelevação na redução da severidade de acidentes (Sameen e Pradhan 2016). Outras variáveis encontradas como relevantes 
nesse caso, na medida em que os valores desses parâmetros aumentam, foram a velocidade no aumento (Renski et al, 1999; Chikkakrishna et al, 2017), a iluminação na redução (De Oña et al, 2011), a condição climática desfavorável no aumento (Anastasopoulos et al., 2012; Ma et al., 2015) e na redução (Castro et al, 2013; Chen et al., 2016; Shrestha e Shrestha, 2017; Milton et al, 2008), a faixa etária elevada no aumento (Chen et al., 2016), o elevado atrito entre pista e veículo na redução (Anastasopoulos et al., 2012), a influência de álcool no aumento (Chen et al., 2016) e a presença de cinto de segurança na redução da severidade de acidentes (Hernandez e Anderson, 2018).

Nesse contexto, este estudo tem o objetivo de identificar os impactos que os parâmetros geométricos causam na frequência e na severidade dos acidentes, assim como os impactos que variáveis ambientais e do usuário causam na severidade de acidentes, ambos para curvas de rodovias de pista simples, com enfoque no primeiro caso. Como objetivo específico, este estudo concilia o objetivo de desenvolver de uma base de dados capaz de associar os registros de acidentes aos parâmetros geométricos da rodovia, obtida pelo processo de reconstituição geométrica. Para isso, este artigo está estruturado em cinco seções, incluindo esta introdução e revisão teórica. A seção 2 descreve o processo de reconstituição geométrica e modelagem estatística, a seção 3 apresenta a construção da base de dados do caso estudado e a seção 4 descreve a estimação dos modelos e a discussão dos resultados obtidos, enquanto que a seção 5 traz as considerações finais do estudo.

\section{MÉTODO}

Este trabalho foi desenvolvido a partir de duas etapas metodológicas principais: (i) construção da base de dados a partir de reconstituição geométrica da rodovia, ajustes quilométricos, processamento dos parâmetros geométricos e compatibilização com os registros de acidentes (DPRF, 2017); e (ii) modelagem da frequência e severidade dos acidentes.

\subsection{Obtenção dos parâmetros geométricos}

Foram adotados conceitos de projeto de rodovias para reconstituir o projeto do trecho rodoviário analisado em formato digital, de modo a ser possível a extração dos parâmetros geométricos. Em seguida foi realizado o ajuste das quilometragens resultantes da reconstituição geométrica aos marcos quilômetros existentes ao longo da rodovia. Por fim, obtida a classe e relevo da rodovia, pode-se extrair os parâmetros geométricos.

\subsubsection{Reconstituição geométrica}

A reconstituição geométrica permite a obtenção de elementos planialtimétricos da rodovia. A rodovia a ser reconstituída é dividida em trechos conforme informações do Sistema Nacional de Viação (SNV) vigente (BRASIL, 2011), de modo a simplificar o processo e reduzir o poder de processamento necessário. Para esse processo, é necessária uma base de dados de pontos com coordenadas georreferenciadas, com precisão suficiente para a identificação das curvas de concordância horizontais e verticais.

A Figura 1 ilustra as etapas do processo de reconstituição geométrica, que se inicia com a importação dos pontos de coordenadas para o software SAEPRO (Sistema Avançado para Estudos e Projeto Viários) [versão 1.0.0.26]. Cada umas das linhas de pontos disposta na etapa 1 representa uma faixa de rolamento da rodovia, percorrida pelo veículo que realizou a coleta dos pontos por meio de equipamento GPS. Na etapa 2, a partir dos pontos disponíveis, é obtido para 
os segmentos em tangente, retas que melhor se adaptam a esses pontos. A partir dessas retas é obtido o eixo da rodovia no trecho em questão, e a partir desse, as curvas de concordância horizontais.

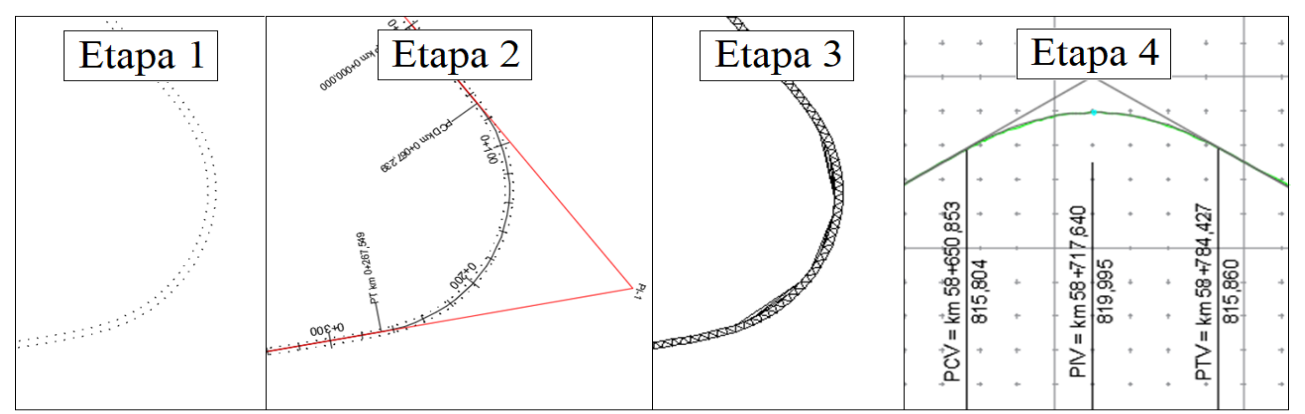

Figura 1. Etapas do processo de reconstituição geométrica

Na etapa 3 é obtida a superfície do terreno, necessária para a reconstituição altimétrica. Essa superfície é recomposta pelo processamento da geometria pelo SAEPRO, mediante a criação de uma malha irregular triangularizada. A superfície obtida representa a real configuração da rodovia, de modo que a definição do perfil altimétrico da rodovia, na etapa 4, deve buscar o melhor ajuste possível com o terreno obtido.

\subsubsection{Ajustes quilométricos}

Tendo em vista que a coleta da localização dos acidentes indicada no registro de acidentes, até o ano de 2017, era realizada apenas com base nos marcos quilométricos existentes ao longo da rodovia, é necessário verificar os valores de quilometragem resultantes da reconstituição geométrica em comparação com a localização dos marcos quilômetros. Para esse processo é utilizada a ferramenta Street View (Google Earth), para localizar os marcos quilométricos mais próximos do início e fim de cada trecho, e o software SAEPRO para realizar o ajuste. Caso os valores divirjam, esses devem ser corrigidos conforme Figura 2 onde o valor da quilometragem inicial do trecho é alterada de modo que a posição do marco quilométrico coincida com a quilometragem no mesmo local.

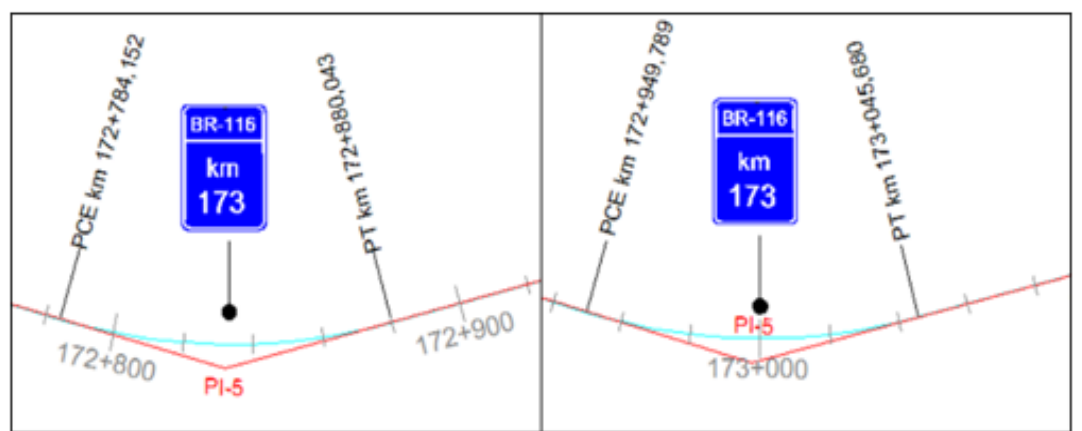

Figura 2. Exemplo de correção do erro do SNV em relação ao marco quilométrico

\subsubsection{Extração e filtragem dos parâmetros}

Os parâmetros geométricos necessários para as análises propostas são obtidos por meio dos relatórios de planimetria (RPL) e altimetria (RAT) gerados pelo SAEPRO. O Ângulo Central, Desenvolvimento e Raio são obtidos diretamente do RPL, já a Superelevação máxima para a classe 
e relevo da rodovia $\left(e_{\max }\right)$ pode ser obtida a partir dos raios fornecidos pelo RPL, quando aplicados na equação (1).

$$
e=e_{\text {max }} \times\left(\frac{2 \times R_{\min }}{R}-\frac{R_{\min ^{2}}}{R^{2}}\right)
$$

em que $R_{\min }$ é o raio mínimo para determinada classe e relevo e $R$ é o raio da curva. A aplicação dessa equação é condicionada à determinação da classe e relevo dos trechos da rodovia, que são obtidos analisando o raio mínimo, constante k mínima e declividade máxima existente para cada trecho analisado. A Declividade de acidentes que ocorram em rampa é obtida diretamente do RAT, enquanto para segmentos em curva de concordância vertical, simplifica-se esta por meio de duas rampas, de modo a obter valores pontuais de declividade.

\subsection{Modelagem estatística}

Nesta seção estão dispostos os procedimentos metodológicos para a estimação dos modelos econométricos para a frequência e a severidade de acidentes, pela aplicação do modelo Binomial Negativo e Logit Ordenado, respectivamente. 0 primeiro passo no processo de modelagem é a definição das variáveis candidatas aos modelos, selecionadas a partir da revisão da literatura. A partir da definição das variáveis candidatas é necessário verificar a existência de multicolinearidade, que ocorre quando algumas variáveis independentes compartilham variância com outras variáveis independentes. A multicolinearidade foi avaliada pelo fator de inflação da variância (VIF), que traduz o valor de tolerância, o qual expressa diretamente o grau de multicolinearidade em um impacto sobre o processo de estimação. Um valor de VIF igual a 1 indica a inexistência de multicolinearidade e valores de VIF maiores que 10 indicam multicolinearidade elevada, geralmente utilizados como valores de corte (Hair et al., 2009).

\subsubsection{Modelo de frequência de acidentes}

Para a estimação do modelo de frequência de acidentes, foi necessário identificar o modelo que mais se adeque para os dados disponíveis. Quando a distribuição de frequência dos acidentes não verifica as hipóteses de normalidade, demonstrando assimetria na distribuição, analisa-se a dispersão da distribuição (Mccullangh e Nelder, 1989). A análise da superdispersão deve ser realizada, e se confirmada a hipótese de média inferior à variância, é indicada a aplicação do modelo Binomial Negativo (Greene, 2012). 0 modelo é expresso pela generalização do modelo de Poisson, em que o termo do erro é dado pela distribuição Gamma - considerando que $u_{i}=\exp \left(\varepsilon_{i}\right)$ - conforme demonstra a equação (2).

$$
\begin{array}{lll} 
& & \ln \left(\mu_{i}\right)=\sum_{j} \beta_{j} x_{i j}+\varepsilon_{i}=\ln \left(\lambda_{i}\right)+\ln \left(u_{i}\right) \\
\text { onde: } & \mu_{i} & \text { média da distribuição; } \\
& x_{i j} & \text { variáveis explicativas; } \\
& \beta_{j} & \text { parâmetros estimados; e } \\
& \varepsilon_{i} & \text { erro de especificação. }
\end{array}
$$

A função de ligação é dada pela distribuição de Poisson, na qual $\lambda_{i}$ é o número esperado de acidentes, conforme equação (3):

$$
\lambda_{i}=\exp \left(\sum_{j} \beta_{j} x_{i j}\right)
$$

A estimação do modelo foi realizada no software IBM SPSS Statistics 23, utilizando a técnica de eliminação regressiva (backward elimination). 0 ajuste do modelo foi avaliado pelo Akaike Information Criterion (AIC) (Akaike, 1992), que permite comparar diferentes modelos pelo equilíbrio entre o viés e a variância explicada pelos modelos. Assim, o modelo mais equilibrado 
é aquele que apresenta o menor AIC. Também foi avaliado a razão pela razão entre desviância e graus de liberdade (Colin e Pravin, 2013; Hilbe, 2014), para o qual valores próximos a 1 indicam melhor ajuste do modelo estimado.

Apesar do modelo Binomial Negativo permitir identificar as variáveis significativas e suas influências na frequência de acidentes, a diferença nas escalas das variáveis pode dificultar a compreensão dos resultados. A comparação da magnitude do impacto de cada variável no respectivo modelo pode ser obtida pelo cálculo do efeito marginal (Hilbe, 2011), dado pela Equação 4, sendo que o incremento das variáveis contínuas foi estabelecido como $10 \%$ da média de todos os valores existentes de cada uma.

$$
\partial E\left(y \mid x_{i j}\right) / \partial x=\beta_{i j}\left[\exp \left(x_{i j} \beta_{j}\right)\right]
$$

em que $x_{i j}$ é variável explicativa, $y$ é a variável dependente e $\beta$ é o parâmetro estimado.

\subsubsection{Modelo de severidade de acidentes}

Para a construção do modelo de severidade de acidentes, foi estimado o modelo Logit Ordenado, o qual é um modelo de escolha discreta, em que a variável dependente severidade é discreta e seus valores traduzem uma ordenação (Small, 1987). 0 modelo assume que a variável latente $y_{i}^{*}$ é uma combinação linear de um conjunto de variáveis explicativas (Greene, 2012), em que os parâmetros $\beta^{\prime}$ são estimados pelo método de máxima verossimilhança, que busca maximizar a função:

onde: $\quad y_{i}^{*} \quad$ variável dependente;

$$
y_{i}^{*}=\beta^{\prime} x_{i}+\varepsilon_{i}
$$

$x \quad$ vetor de atributos observáveis que determinam a ordem discreta de cada evento;

$\beta^{\prime} \quad$ vetor de parâmetros estimados; e

$\varepsilon_{i} \quad$ erro de especificação.

A variável dependente severidade $\left(y_{i}\right)$ assume os valores de severidade 1, 2, 3 e 4 para danos materiais, feridos leves, feridos graves e vítimas fatais, respectivamente. 0 termo não observado $\left(\varepsilon_{i}\right)$, ou componente aleatória, é dado pela distribuição logística. No modelo Logit Ordenado estima-se a probabilidade generalizada do acidente estar em determinada categoria $m$, conforme:

onde: L distribuição logística acumulada;

$$
\begin{gathered}
P\left(y_{i}=m\right)=L\left(\mu_{m}-\beta^{\prime} x_{i}\right)-L\left(\mu_{m-1}-\beta^{\prime} x_{i}\right) \\
L\left(\beta^{\prime} x\right) \frac{e^{\beta^{\prime} x_{i}}}{1+e^{\beta^{\prime} x_{i}}}
\end{gathered}
$$

$\mu_{m}$ pontos de corte;

$x \quad$ vetor de atributos observáveis que determinam a ordem discreta de cada evento;

$\beta^{\prime} \quad$ vetor de parâmetros estimados.

0 modelo Logit ordenado foi estimado por eliminação regressiva (backward elimination) no software Biogeme (Bierlaire, 2003). 0 ajuste geral do modelo foi avaliado através do teste da razão de verossimilhança, que demonstra que o modelo obtido é estatisticamente significativo (p-valor $<0,05$ ), para o nível de significância de 5\%, que indica que a variável independente é significativa ao nível de confiança de $95 \%$. A medida de ajuste geral do modelo é expressa pelo valor de Pseudo- $\mathrm{R}^{2}$ (entre 0 e 1, em que 1 significa ajuste exato), no qual valores próximos a 0,4 podem ser considerados bons ajustes (Ortúzar e Willumsen, 2011). 
O sinal dos coeficientes estimados para as variáveis indica a impacto positivo ou negativo na severidade dos acidentes. Porém, no modelo Logit ordenado, o cálculo de efeitos marginais é estritamente necessário para a compreensão do efeito das estimativas dos parâmetros individuais. Nesse modelo, o efeito marginal da probabilidade de um acidente ter uma severidade $m$ em relação a uma variável $x$ foi calculado pela Equação 8 (Greene, 2012):

$$
P\left(y_{i}=m\right) / \partial x=\left[L\left(\mu_{m}-\beta^{\prime} x_{i}\right)-L\left(\mu_{m-1}-\beta^{\prime} x_{i}\right)\right] \beta
$$

Para as variáveis contínuas o efeito foi calculado pelo incremento de $10 \%$ da média das observações, e para as variáveis discretas pelo incremento de uma unidade. Para variáveis dummy, tal relação não faria sentido (Greene, 2012), assim, o efeito marginal foi calculado como a diferença de probabilidade para $x_{i}$, (que é a variável dummy) assumindo os valores 0 e 1 , conforme Equação 9:

$$
x_{i}=P\left(y_{i}=m\right)\left|x_{i}=1-P(y=m)\right| x_{i}=0
$$

"Valores positivos de $\beta^{\prime}$ indicam que um aumento em implicara no aumento da probabilidade da severidade mais alta, analogamente, valores positivos de $\beta^{\prime}$ indicam o aumento da probabilidade de ocorrer a severidade de nível mais baixo.", se possível trocar o segundo positivo por "negativo", ficando: "Valores positivos de $\beta^{\prime}$ indicam que um aumento em implicara no aumento da probabilidade da severidade mais alta, analogamente, valores negativos de $\beta^{\prime}$ indicam o aumento da probabilidade de ocorrer a severidade de nível mais baixo."

\section{CONSTRUÇÃO DA BASE DE DADOS}

Foram considerados neste estudo trechos de pista simples da BR 116 no Rio Grande do Sul, do quilômetro 0 ao 235. Os acidentes considerados foram capotamento, tombamento, colisão com objeto fixo e saída de pista. Um fato que corrobora essa hipótese é a ocorrência, em curvas dos trechos estudados, de acidentes dos tipos considerados em 72,6\% dos casos, contra 30,9\% para os demais tipos de acidentes. Foram analisados acidentes entre os anos de 2007 e 2016, considerando que não ocorreram mudanças significativas na geometria da rodovia estudada.

Os segmentos desse estudo foram reconstituídos por meio de um levantamento de coordenadas que incluía toda a extensão da BR-116 no estado do Rio Grande do Sul, onde a partir desse universo limitou-se o estudo para segmentos de pista simples e região montanhosa, de modo a realizar as análises em segmentos mais homogêneos possíveis. Essa delimitação resultou em 12 trechos de pista simples, com um total de 225 quilômetros. Nesse universo foram observados 2007 acidentes em curva dos tipos saída de pista, tombamento, capotamento e colisão com objeto fixo. Os segmentos foram então divididos em trechos, conforme SNV vigente.

O processo de reconstituição geométrica resultou, após simplificações, em trechos de Classe III e região Montanhosa (DNIT, 1999), com 729 curvas de concordância horizontal. Após realizado o cruzamento das informações dessa reconstituição com as informações de acidentes, foram obtidos, para cada um dos 2.007 acidentes considerados, sua localização, curva, parâmetros geométricos e outras características disponíveis na base de dados da PRF. A distribuição de frequência dos acidentes em curvas é apresentada na figura 3.

Nos modelos de frequência de acidentes foram utilizadas como variáveis os parâmetros geométricos extraídos da base de dados construída, conforme Tabela 1. Tendo em vista que foi construído um modelo de frequência de acidentes em curvas, não foi possível incluir as características individuais dos acidentes disponibilizadas na base de dados da PRF. De modo a incluir a informação sobre a ocorrência do acidente em aclive ou declive, que também é particular do 
acidente, foram construídos modelos de frequência de acidentes em aclive e declive, além do modelo de frequência de acidentes totais, que considera a declividade absoluta.

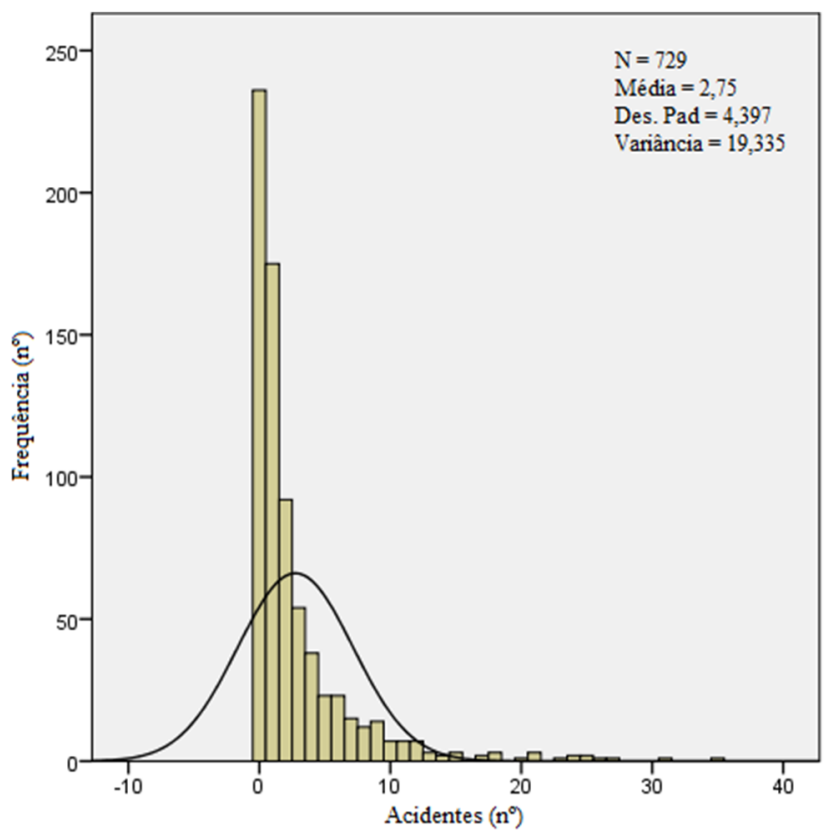

Figura 3. Distribuição de frequência dos acidentes em curvas

Tabela 1 - Estatística descritiva para os modelos de frequência

\begin{tabular}{lllllll}
\hline Variável & & N & Mínimo & Máximo & Média & Desvio padrão \\
\hline Dependente & Frequência de acidentes ( $\mathrm{n}$ - $)$ & 729 & 0,000 & 35,000 & 2,753 & 4,397 \\
\hline \multirow{4}{*}{ Independentes } & 729 & 0,370 & 164,669 & 44,086 & 31,722 \\
& Ângulo Central $\left(^{\circ}\right)$ & 729 & 0,020 & 9,908 & 4,451 & 2,259 \\
& Declividade Absoluta (\%) & 729 & 0,646 & 427,960 & 108,116 & 59,788 \\
& Desenvolvimento (m) & 729 & 45,420 & 5490,900 & 202,447 & 263,469 \\
& Raio (m) & 729 & 2,000 & 8,000 & 4,657 & 1,769 \\
& Superelevação (\%) & & & & & \\
\hline
\end{tabular}

O modelo de severidade de acidentes, pela natureza desagregada de sua análise, permitiu a inclusão das variáveis ambientais e do usuário disponíveis na base de dados da PRF, conforme Tabela 2. Além das variáveis utilizadas no modelo de frequência de acidentes, foram adicionadas as variáveis dummy Aclive, Chuva, Dia, Neblina e Velocidade Incompatível.

Tabela 2 - Tabela de frequências para as variáveis adicionadas ao modelo de severidade

\begin{tabular}{llllll}
\hline Variável & & Severidade 1 & Severidade 2 & Severidade 3 & Severidade 4 \\
\hline Dependente & Severidade (0-4) & $1153(59,5 \%)$ & $588(30,3 \%)$ & $147(7,6 \%)$ & $50(2,6 \%)$ \\
\hline \multirow{5}{*}{ Independentes } & Aclive $(0 ; 1)$ & $441(60,3 \%)$ & $230(31,5 \%)$ & $45(6,3 \%)$ & $15(2,1 \%)$ \\
& Chuva (0;1) & $490(77,0 \%)$ & $118(18,6 \%)$ & $19(3,0 \%)$ & $9(1,4 \%)$ \\
& Dia $(0 ; 1)$ & $696(57,9 \%)$ & $383(31,8 \%)$ & $98(8,1 \%)$ & $26(2,2 \%)$ \\
& Neblina (0;1) & $45(68,2 \%)$ & $11(16,7 \%)$ & $6(9,1 \%)$ & $4(6,1 \%)$ \\
& Velocidade Incompatível (0;1) & $530(57,2 \%)$ & $278(30,0 \%)$ & $84(9,1 \%)$ & $35(3,8 \%)$ \\
\hline
\end{tabular}

As variáveis dummy assumem valores de 0 e 1, de modo a indicar a presença ou ausência de determinada característica. As variáveis Chuva e Neblina indicam a condição climática no momento do acidente, a variável Dia indica se o acidente ocorreu de dia ou à noite, e a variável Velocidade Incompatível indica se o motorista estava com uma velocidade incompatível com a velocidade máxima do local em questão. 


\section{RESULTADOS}

Nesta seção são apresentados os resultados da construção dos modelos de frequência de acidentes e do modelo de severidade de acidentes. Através da aplicação do teste do fator de inflação da variância (Variance Inflation Factor - VIF) foi descartada a presença de variáveis multicolineares em qualquer um dos modelos, que podem implicar na dificuldade de estimação e de interpretação dos modelos por compartilharem variância. Assim, o valor de VIF máximo tolerado foi de 10 (Hair et al., 2009).

\subsection{Modelos de frequência de acidentes}

Foram estimados modelos Binomiais Negativos para a frequência de acidentes totais em curvas, frequência de acidentes em curvas com aclives e frequência de acidentes em curvas com declives, de modo a incluir na análise a informação sobre a ocorrência do acidente em aclive ou declive. A técnica de eliminação regressiva (backwards elimination) foi aplicada para um nível de significância de 10\%, de modo a incluir a variável Superelevação na análise do modelo de frequência de acidentes em declives e as variáveis Declividade Absoluta e Aclive na análise do modelo de severidade de acidentes. Esse procedimento resultou nas variáveis dispostas na Tabela 3 , onde coeficientes positivos indicam que a variável aumenta a frequência de acidentes, na medida que seu valor aumenta. Na tabela 4 estão dispostos os efeitos marginais, que permitem uma melhor compreensão dos resultados, tendo em vista as diferentes grandezas das variáveis.

Tabela 3 - Modelo binomial negativo para a frequência de acidentes

\begin{tabular}{lcccccc}
\hline \multirow{2}{*}{ Variável } & \multicolumn{2}{c}{ Acidentes Totais } & \multicolumn{2}{c}{ Acidentes em Aclive } & \multicolumn{2}{c}{ Acidentes em declive } \\
\cline { 2 - 6 } & Coeficiente & Valor $\mathbf{p}$ & Coeficiente & Valor $\mathbf{p}$ & Coeficiente & Valor $\mathbf{p}$ \\
\hline (Intercepto) & $-0,679$ & 0,001 & $-1,689$ & 0,000 & $-0,949$ & 0,000 \\
Ângulo Central ( ${ }^{\circ}$ ) & & & 0,009 & 0,029 & 0,013 & 0,000 \\
Declividade Absoluta (\%) & 0,075 & 0,000 & & & & 0,000 \\
Desenvolvimento (m) & 0,005 & 0,000 & 0,005 & 0,008 & 0,101 & $0,079 *$ \\
Superelevação (\%) & 0,161 & 0,000 & 0,138 & 0,041 & 0,065 & 1,16 \\
Deviance/df & & 1,32 & & 1,073 & & 2475,33 \\
AIC & & 3099,84 & & 1928,164 & & \\
\hline
\end{tabular}

Tabela 4 - Efeitos marginais para os modelos de frequência de acidentes

\begin{tabular}{lcccccc}
\hline \multirow{2}{*}{ Variável } & \multicolumn{2}{c}{ Acidentes Totais } & \multicolumn{2}{c}{ Acidentes em Aclive } & \multicolumn{2}{c}{ Acidentes em declive } \\
\cline { 2 - 7 } & Incremento & $\begin{array}{c}\text { Efeito } \\
\text { marginal }\end{array}$ & Incremento & $\begin{array}{c}\text { Efeito } \\
\text { marginal }\end{array}$ & Incremento & $\begin{array}{c}\text { Efeito } \\
\text { marginal }\end{array}$ \\
\hline Ângulo Central $\left(^{\circ}\right.$ ) & 0,445 & 0,338 & 4,409 & 0,403 & 4,409 & 0,602 \\
Declividade Absoluta (\%) & 10,812 & 0,55 & 10,812 & 0,517 & 0,445 & 0,461 \\
Desenvolvimento (m) & 0,466 & 0,777 & 0,466 & 0,663 & 0,466 & 0,309 \\
Superelevação (\%) & & & & & & \\
\hline
\end{tabular}

\subsubsection{Modelo de frequência de acidentes totais}

No modelo estimado para a frequência de acidentes totais, foram encontradas como variáveis significativas a Declividade Absoluta, o Desenvolvimento e a Superelevação. Os resultados mostraram a influência dessas variáveis no sentido de aumentar a frequência de acidentes, corroborando com os resultados da literatura.

A Declividade Absoluta se demonstrou significativa para o aumento na frequência de acidentes, à medida que ela aumenta, conforme resultados da literatura (Shankar et al, 1995; Agbelie, 2016b). Esse resultado pode ser explicado pela maior dificuldade que regiões de grande 
declividade apresentam ao motorista e pela redução da margem de segurança do atrito transversal, que contribuem para a saída de pista do veículo (Torbic et al., 2014).

A análise dos efeitos marginais permitiu concluir que o Desenvolvimento possui maior influência na frequência de acidentes do que a Declividade Absoluta, aumentando a frequência de acidentes com o seu aumento, conforme resultados da literatura (Aram, 2010; Schneider et al, 2010). É possível interpretar esse resultado através de um maior tempo de permanência do motorista na curva, pois o desenvolvimento representa o percurso que o veículo percorre na curva horizontal. Apesar disso, um maior Desenvolvimento pode significar maior Raio, maior Ângulo Central ou ambos.

A terceira variável obtida como significativa foi a superelevação, que segundo os resultados dos efeitos marginais foi a de maior impacto, possuindo mais que o dobro de influência na frequência de acidentes. Apesar do resultado não é possível concluir que a superelevação está diretamente associada a uma maior frequência de acidentes, pois ela possui maior valor em locais mais críticos, com menor Raio, o que explica o resultado. No entanto, esse resultado vai de encontro com o obtido na literatura (Aram, 2010; Garniak, 2014).

\subsubsection{Modelo de frequência de acidentes em aclives}

Para o modelo estimado, levando em conta apenas os acidentes ocorridos em aclive, as variáveis significativas foram Ângulo Central, Desenvolvimento e Superelevação. A variável Declividade não se demonstrou um fator significativo. Uma explicação para tal resultado é a ocorrência de dois efeitos adversos com o aumento da declividade: o acréscimo da perda de atrito transversal (Torbic et al., 2014) e a redução da velocidade do veículo pelo condutor (Garcia et al, 2013).

o Ângulo Central foi a única variável significativa nesse modelo que não se mostrou significativa no modelo anterior. Segundo os resultados de efeitos marginais a frequência de acidentes aumenta com o aumento do Ângulo Central, o que é intuitivo e se confirma na literatura (Aram, 2010; Garniak, 2014; Dong et al., 2015; Rengarasu et al, 2009), pois um maior Ângulo Central significa curvas mais acentuadas. As variáveis Desenvolvimento e Superelevação já foram discutidas no modelo anterior.

\subsubsection{Modelo de Frequência de acidentes em declives}

Considerando o modelo de frequência de acidentes apenas em declive, as variáveis significativas foram Ângulo Central, Declividade e Superelevação. A variável Ângulo Central se demonstrou mais significativa do que a variável Superelevação, diferente do observado no modelo de frequência de acidentes em aclives. A variável Declividade, ao contrário do observado no modelo de frequência de acidentes em aclive, foi significativa. Esse resultado pode ser explicado pelo fato dos declives serem mais críticos do que aclives, conforme resultados da literatura (Bonneson, 2000; Eck e French, 2002).

\subsection{Modelo de severidade de acidentes}

A estimação do modelo de severidade de acidentes, assim como para a frequência de acidentes, foi realizada por eliminação regressiva. Após a aplicação dessa técnica, as variáveis Raio, Dia e Desenvolvimento, respectivamente, não se demonstraram dentro do nível de confiança de 90\%. As variáveis significativas e seus efeitos marginais são apresentadas pelas Tabela 5 e 6 respectivamente: 
Tabela 5 - Modelo Logit Ordenado para a severidade de acidentes

\begin{tabular}{llll}
\hline Variável & Coeficiente & Teste t & Valor $\mathbf{p}$ \\
\hline Ângulo Central $\left({ }^{\circ}\right)$ & 0,004 & 2,47 & 0,01 \\
Declividade Absoluta $(\%)$ & $-0,035$ & $-1,73$ & $0,080^{*}$ \\
Superelevação (\%) & $-0,105$ & $-2,73$ & 0,01 \\
Aclive $(0 ; 1)$ & $-0,165$ & $-1,71$ & $0,090^{*}$ \\
Chuva $(0 ; 1)$ & $-1,23$ & $-11,12$ & 0 \\
Neblina $(0 ; 1)$ & $-0,618$ & $-2,26$ & 0,02 \\
\hline
\end{tabular}

Tabela 5 - Modelo Logit Ordenado para a severidade de acidentes (cont.)

\begin{tabular}{llll}
\hline Variável & Coeficiente & Teste $\mathbf{t}$ & Valor $\mathbf{p}$ \\
\hline Velocidade Incompatível $(0 ; 1)$ & 0,357 & 3,81 & 0 \\
tau1 & $-0,353$ & $-1,83$ & 0,07 \\
tau2 & 1,54 & 7,71 & 0 \\
tau3 & 3,01 & 12,89 & 0 \\
Pseudo- $R^{2}$ & & & 0.337 \\
Log-likelihood & & & $-1779,91$ \\
AlC & & $-3545,81$ \\
\hline * & &
\end{tabular}

*Valores significativos em um nível de confiança de $90 \%$

Tabela 6 - Efeitos marginais para o modelo de severidade de acidentes

\begin{tabular}{lllcrr}
\hline Variável & Incremento & $\begin{array}{l}\text { Efeito marginal } \\
\text { (Severidade 1) }\end{array}$ & $\begin{array}{l}\text { Efeito marginal } \\
\text { (Severidade 2) }\end{array}$ & $\begin{array}{r}\text { Efeito marginal } \\
\text { (Severidade 3) }\end{array}$ & $\begin{array}{c}\text { Efeito marginal } \\
\text { (Severidade 4) }\end{array}$ \\
\hline Ângulo Central $\left({ }^{\circ}\right)$ & 6,2180 & $-0,00094$ & 0,00056 & 0,00027 & 0,00011 \\
Declividade Absoluta (\%) & 0,4738 & 0,00783 & $-0,00472$ & $-0,00224$ & $-0,00088$ \\
Superelevação (\%) & 0,5341 & 0,02323 & $-0,01409$ & $-0,00658$ & $-0,00256$ \\
Aclive (0;1) & 1 & 0,03655 & $-0,02211$ & $-0,0104$ & $-0,00405$ \\
Chuva (0;1) & 1 & 0,19352 & $-0,12902$ & $-0,04724$ & $-0,01726$ \\
Neblina (0;1) & 1 & 0,13774 & $-0,08987$ & $-0,03489$ & $-0,01297$ \\
Velocidade Incompatível (0;1) & 1 & $-0,02805$ & 0,0165 & 0,0083 & 0,00326 \\
\hline
\end{tabular}

O aumento da variável Ângulo Central produz, segundo a análise dos efeitos marginais, uma pequena redução na probabilidade de ocorrência da severidade 1 (danos materiais) e um pequeno aumento na probabilidade de ocorrência das severidades 2, 3 e 4 (feridos leves, feridos graves e fatais, respectivamente). Esse resultado pode ser explicado com ajuda dos resultados dos modelos de frequência de acidentes em aclive e declive, onde o Ângulo Central teve influência no aumento da frequência dos acidentes.

A variável Declividade também apresenta pequeno efeito na severidade de acidentes, mas apesar de um aumento na Declividade provocar um aumento na frequência de acidentes, a análise dos efeitos marginais do modelo de severidade de acidentes mostra um aumento na probabilidade de ocorrência da severidade 1 e uma redução da probabilidade de ocorrência das severidades 2, 3 e 4, de acordo com resultados da literatura (Chikkakrishna et al, 2017), e pode ser explicado pela tendência de redução da velocidade operacional que ocorre em elevadas declividades (Garcia et al, 2013), que por sua vez leva a acidentes de menor severidade, conforme discutido no final deste capítulo.

O último parâmetro geométrico utilizado nos modelos de severidade de acidentes que se mostrou uma variável significativa foi a Superelevação, que produziu resultados semelhantes ao da variável Declividade, e que podem ser interpretados de mesmo modo. A análise dos efeitos marginais produziu um aumento da probabilidade de ocorrência da severidade 1 e uma redução da probabilidade de ocorrência das severidades 2, 3 e 4, semelhantes aos resultados da literatura (Sameen e Pradhan, 2016). A variável Superelevação depende do Raio da rodovia, sendo que uma maior Superelevação ocorre em Raios pequenos, que por sua vez produz velocidades operacionais reduzidas e acidentes de menor severidade. 
A variável dummy Aclive é importante para um melhor entendimento da variável declividade e por depender do acidente só pode ser analisada no modelo de severidade. Os resultados indicam que a ocorrência de um acidente em aclive aumenta em 3,6\% a probabilidade de acidentes de severidade 1 e que a ocorrência de um acidente em declive aumenta a probabilidade de acidentes das severidades 2, 3 e 4 em 2,2\%, 1,0\% e 0,4\%, respectivamente. Esse resultado vai de encontro com os resultados observados nos modelos de frequência de acidentes, onde a variável declividade se mostrou significativa no modelo de frequência de acidentes em declives, que são mais críticos segundo literatura (Bonneson, 2000; Eck e French 2002).

As variáveis dummy Chuva e Neblina são ambas variáveis ambientais que produziram um efeito marginal considerável no sentido de aumentar a probabilidade de acidentes de severidade 1 e reduzir a probabilidade de acidentes de severidades 2, 3 e 4, que está de acordo com resultados da literatura (Anastasopoulos et al., 2012; Ma et al., 2015). Esse comportamento se explica pela diminuição do nível de conforto que o motorista percebe ao trafegar na chuva ou neblina, que o faz reduzir a velocidade, e por consequência, a severidade do acidente.

A última variável que se mostrou significativa no modelo de severidade foi Velocidade Incompatível, que produziu uma redução na probabilidade de ocorrência da Severidade 1 um aumento na probabilidade de ocorrência das severidades 2, 3 e 4. Esse resultado corrobora resultados da literatura, onde uma maior velocidade leva a severidades mais elevadas (Renski et al, 1999; Chikkakrishna et al, 2017; Ma et al., 2015).

\section{CONSIDERAÇÕES FINAIS}

Este trabalho buscou analisar o impacto de parâmetros geométricos de curvas na frequência e severidade de acidentes em rodovias de pista simples. Além das variáveis geométricas, foram incluídas na análise as variáveis relacionadas a fatores humanos (o desempenho de Velocidade Incompatível) e variáveis ambientais (Chuva, Dia, Neblina). Estimou-se os modelos econométricos Binomial Negativo e Logit Ordenado, nos quais, dentre os cinco parâmetros geométricos analisados: Ângulo Central, Declividade Absoluta, Desenvolvimento, Raio e Superelevação, apenas a variável Raio não foi significativa em nenhum dos modelos. Esse resultado, no entanto, é esperado pela relação existente entre as variáveis Superelevação e Raio, tendo em vista que a região de estudo foi considerada de classe uniforme. Entre as variáveis significativas, a Declividade absoluta e a Superelevação foram consistentemente associadas ao aumento da frequência e à redução da severidade dos acidentes.

Os resultados obtidos indicam que os locais que dispõem de parâmetros de projeto com valores críticos parecem estar associados a uma maior frequência e menor severidade de acidentes, como o caso da superelevação. Essa está presente em curvas com menor raio, locais que se tornam críticos na presença de velocidade acima da regulamentada. Assim, maiores superelevações podem permitir a minimização dos acidentes que possam ocorrer. Nesse sentido, podese inferir que a segurança que a rodovia efetivamente oferece e a percepção de segurança do usuário nem sempre estão alinhados. Portanto, sugere-se a aplicação de medidas que aproximem esses dois conceitos. Abordagens como análises de consistência geométrica e inclusão de variáveis comportamentais em conjunto com o desenvolvimento de modelos de frequência e severidade de acidentes tem o potencial de contribuir com o entendimento da acidentalidade em curvas. Além disso, a análise da frequência e severidade de acidentes a partir de parâmetros geométricos seja replicada em rodovias de pista dupla. Em relação à modelagem da frequência 
e severidade, a análise utilizando modelos de parâmetros mistos pode contribuir com a acurácia da estimação.

\section{REFERÊNCIAS BIBLIOGRÁFICAS}

AASHTO (2011) A Policy on Geometric Design of Highways and Streets. American Association Of State Highway And Transportation Officials. (AASTHO) 5th ed. Washington, D.C.

Agbelie, B. R. D. K. (2016a) A comparative empirical analysis of statistical models for evaluating highway segment crash frequency. Journal of Traffic and Transportation Engineering, v. 3, n. 4, p. 374-379. DOI: 10.1016/j.jtte.2016.07.001

Agbelie, B. R. D. K. (2016b) Random-parameters analysis of highway characteristics on crash frequency and injury severity. Journal of Traffic and Transportation Engineering, v. 3, n. 3, p. 236-242. DOI: 10.1016/j.jtte.2015.09.006

Akaike H. (1992) Information Theory and an Extension of the Maximum Likelihood Principle. In: Kotz S. e N. L. Johnson (eds) Breakthroughs in Statistics. Springer Series in Statistics (Perspectives in Statistics). Springer, New York, NY. DOI: 10.1007/978-1-4612-0919-5_38

Anastasopoulos, P. C.; V. N. Shankar; J. E. Haddockc e F. L. Mannering (2012) A multivariate tobit analysis of highway accidentinjury-severity rates. Accident Analysis \& Prevention, v. 45, p. 110-119. DOI: 10.1016/j.aap.2011.11.006

Aram, A. (2010) Effective Safety Factors on Horizontal Curves of Two-lane Highways. Journal of Applied Sciences, v. 10, n. 22, p. 2814-2822. DOI: 10.3923/jas.2010.2814.2822

Bierlaire, M. (2003). Biogeme: a free package for the estimation of discrete choice models. Proceedings of the 3rd Swiss Transportation Research Conference, Ascona, Switzerland.

Bonneson, J. A. (2000) Superelevation distribution methods and transition designs. NCHRP Report 439, Transportation Research Board, Washington, DC, USA.

Brasil. (2011) Lei n. ${ }^{\circ}$ 13.379, de 6 de janeiro de 2011. Dispõe sobre o Sistema Nacional de Viação - SNV. Diário Oficial [da] Republica Federativa do Brasil. Brasília, DF.

Castro, M.; R. Paleti e C.R. Bhat (2013) A spatial generalized ordered response model to examine highway crash injury severity. Accident Analysis \& Prevention, v. 52, p. 188-203. DOI: 10.1016/j.aap.2012.12.009

Chen, C.; G. Zhanga; H. Huang; J. Wangc e R. A. Tarefder (2016) Examining driver injury severity outcomes in rural non-interstate roadway crashes using a hierarchical ordered logit model. Accident Analysis \& Prevention, v. 96, p. 79-87. DOI: 10.1016/j.aap.2016.06.015

Chikkakrishna, N. K.; M. Parida e S. S. Jain (2017) Identifying safety factors associated with crash frequency and severity on nonurban four-lane highway stretch in India. Journal of Transportation Safety \& Security, v. 9, n. sup1, p. 6-32, 30. DOI: 10.1080/19439962.2016.1150927

Colin, C. A., e Pravin, T. (2013) Regression analysis of count data, Second edition. Regression Analysis of Count Data, Second Edition. doi:10.1017/CB09781139013567

Cruz, P.; T. Echaveguren e P. González (2017) Estimación del potencial de rollover de vehículos pesados usando principios de confiabilidad. Revista ingeniería de construcción, v. 32, n. 1, p. 5-14. DOI: 10.4067/S0718-50732017000100001

De Oña, J.; R. O. Mujalli e F. J. Calvo (2011) Analysis of traffic accident injury severity on Spanish rural highways using Bayesian networks. Accident Analysis \& Prevention, v. 43, n. 1, p. 402-411. DOI: 10.1016/j.aap.2010.09.010

DNER (1999) Manual de Projeto Geométrico de Rodovias Rurais. Departamento Nacional das Estradas de Rodagem (DNER), Rio de Janeiro, RJ

Dong, C; S. S. Nambisan; S. H. Richards e Z. Ma (2015) Assessment of the effects of highway geometric design features on the frequency of truck involved crashes using bivariate regression. Transportation Research Part A: Policy and Practice, v. 75, p. 30-41. DOI: $10.1016 /$ j.tra.2015.03.007

DPRF (2017) Acidentes. Departamento De Policia Rodoviária Federal (DPRF). Disponível em: <<https://www.prf.gov.br/portal/dados-abertos/acidentes>>. Acesso em: 10 set. 2017

Eck, R. W.; French, L. J. (2002) Effective Superelevation for Large Trucks on Sharp Curves and Steep Grades. West Virginia University, Report 153. Morgantown, WV, USA

Garcia, D. S. P.; L. A. Lindau e C. T. Nodari (2013) Multivariate Operating Speed Forecasting Model Based on the Geometric Elements of Two-Lane Highways. Journal of Traffic and Logistics Engineering, v. 1, n. 2, p. 97-103. DOI: 10.12720/jtle.1.2.97103

Garnaik, M. M. (2014) Effects of Highway geometric elements on Accident Modelling. Thesis (Master of Technology in Transportation Engineering). Department of Civil Engineering, National Institute of Technology Rourkela Odisha, India.

Greene, W. W. H. (2012) Econometric Analysis. 7. ed. Edition. New Jersey: Pearson.

Hair, J. F.; W. C. Black; B. J. Babin; R. E. Anderson e R. L. Tatham (2009) Análise multivariada de dados. Bookman Editora. Hilbe, J. M. (2011) Negative Binomial Regression. 2nd ed. Cambridge University Press. DOI: 10.1017/CBO9780511973420

Hilbe, J. M. (2014) Modelling Count Data. Obtido de www.cambridge.org

Hosseinpour, M.; A. S. Yahaya; A. F. Sadullah; N. Ismail e S. M. R. Ghadiri (2016) Evaluating the effects of road geometry, environment, and traffic volume on rollover crashes. Transport, v. 31, n. 2, p. 221-232. DOI: 10.3846/16484142.2016.1193046

ITF (2016) Zero Road Deaths and Serious Injuries: Leading a Paradigm Shift to a Safe System. International Transport Forum. (ITF). OECD Publishing, Paris. DOI: 10.1787/9789282108055-en 
Iyinam, A. F.; S. Iyinam e M. Ergun (1997) Analysis of relationship between highway safety and road geometric design elements: Turkish case. Technical University of Istanbul, Faculty of Civil Engineering, Turkey.

Ma, J. e Z. Li (2010) Bayesian Modeling of Frequency-Severity Indeterminacy with an Application to Traffic Crashes on TwoLane Highways. American Society of Civil Engineers. Disponível em: <http://ascelibrary.org/doi/10.1061/41127\%28382\%29110>. Acesso em: 24 maio. 2018. DOI: 10.1061/41127(382)110

Ma, Z; W. Zhao; S. I. Chien e C. Dong (2015) Exploring factors contributing to crash injury severity on rural two-lane highways. Journal of Safety Research, v. 55, p. 171-176. 10.1016/j.jsr.2015.09.003

Mannering, F. L. e C. R. Bhat (2014) Analytic methods in accident research analytic methods in accident research: methodological frontier and future directions. Analytic Methods in Accident Research. v. 1, p. 1-22. DOI: 10.1016/j.amar.2013.09.001

Mccullangh, P. e J. A. Nelder (1989) Generalized linear models. 2nd ed. [S.l.]: Chapman and Hall/CRC Monographs on Statistics \& Applied Probability.

Milton, J. C.; V. N. Shankar e F. L. Mannering (2008) Highway accident severities and the mixed logit model: An exploratory empirical analysis. Accident Analysis \& Prevention, v. 40, n. 1, p. 260-266. DOI: 10.1016/j.aap.2007.06.006

Ortúzar, J. D. e L. G. Willumsen (2011) Modelling Transport. 4th ed., London: Wiley. DOI: 10.1109/MITS.2011.2178881

Radimsky, M.; R. Matuszkova e O. Budik (2016) Relationship between horizontal curves design and accident rate. Jurnal Teknologi, v. 78, n. 5-2. DOI: 10.11113/jt.v78.8493

Rengarasu, T.; T. Hagiwara e M. Hirasawa (2009) Effects of Road Geometry and Cross-Section Variables on Traffic Accidents: Study Using Homogeneous Road Segments. Transportation Research Record: Journal of the Transportation Research Board, v. 2102, p. 34-42. DOI: 10.3141/2102-05

Renski, H.; A. Khattak e F. Council (1999) Effect of Speed Limit Increases on Crash Injury Severity: Analysis of Single-Vehicle Crashes on North Carolina Interstate Highways. Transportation Research Record: Journal of the Transportation Research Board, v. 1665, p. 100-108. DOI: 10.3141/1665-14

Sameen, M. I. e B. Pradhan (2016) Forecasting severity of traffic accidents using road geometry extracted from mobile laser scanning data. Conference on Remote Sensing (ACRS).

Schneider, W. H.; P. T. Savolainen e D. N. Moore (2010) Effects of Horizontal Curvature on Single-Vehicle Motorcycle Crashes along Rural Two-Lane Highways. Transportation Research Record: Journal of the Transportation Research Board, v. 2194, n. 1, p. 91-98. DOI: 10.3141/2194-11

Schneider, W. H.; P. T. Savolainen e K. Zimmerman (2009) Driver Injury Severity Resulting from Single-Vehicle Crashes along Horizontal Curves on Rural Two-Lane Highways. Transportation Research Record: Journal of the Transportation Research Board, v. 2102, n. 1, p. 85-92. DOI: 10.3141/2102-11

Shankar, V.; F. Mannering e W. Barfield (1995) Effect of roadway geometrics and environmental factors on rural freeway accident frequencies. Accident Analysis \& Prevention, v. 27, n. 3, p. 371-389. DOI: 10.1016/0001-4575(94)00078-Z

Shrestha, P. P. e K. J. Shrestha (2017) Factors associated with crash severities in built-up areas along rural highways of Nevada: A case study of 11 towns. Journal of Traffic and Transportation Engineering (English Edition), v. 4, n. 1, p. 96-102. DOI: 10.1016/j.jtte.2016.08.003

Small, K.A. (1987) Discrete Choice Model for Ordered Alternatives, Econometrica, v. 55, n. 2, p. 409.

Torbic, D. J; E. T. Donnell; S. N. Brennan; A. Brown; M. K. O'Laughlin e K. M. Bauer (2014) Superelevation Criteria for Sharp Horizontal Curves on Steep Grades. Washington, D.C.: Transportation Research Board. DOI: 10.17226/22312

Treat. J.; N. S. Tumbas; S. T. McDonald; D. Shinar; R. D. Hume; R. E. Mayer; R. L. Stansifer e N. J. Castellan (1979) Tri-level study of the causes of traffic accidents. Executive summary. Vision Research. v. 42, n. 21, p. 2419-2430.

United Nations. (2011) Global Plan for The Decade of Action for Road Safety 2011-2020. Disponível em: www.who.int/roadsafety/decade of action/plan/english.pdf

United Nations. (2017) Sustainable Development Goals. United Nations Department of Public Information. Obtido 20 de junho de 2018, de http://www.undp.org/content/undp/en/home/sustainable-development-goals.html

WHO (2011) Saving Millions of lives: Decade of action for road safety 2011-2020. World Health Organization (WHO). WHO publication (Vol. 11). Obtido de http://who.int/violence_injury_prevention/publications/road_traffic/saving_millions_lives_en.pdf 